\title{
Left Ventricular Assist Device (LVAD) Implantation and Renal Dysfunction
}

\author{
Assem Nogaibayeva ${ }^{1}$, Bagdat Sultanova ${ }^{2}$, Myrzabek Makhmutov ${ }^{3}$
}

${ }^{1}$ Extracorporeal Hemocorrection Laboratory, National Research Cardiac Surgery Center, Astana, Republic of Kazakhstan

${ }^{2}$ Nephrology and Dialysis Department, Kazakh Medical University of Continuing Education, Almaty, Republic of Kazakhstan

${ }^{3}$ Anesthesiology and Intensive Care Department (Adult), National Research Cardiac Surgery Center, Astana, Republic of Kazakhstan

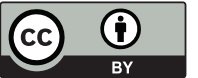

This work is licensed under a Creative Commons Attribution 4.0 International License

Received: 2018-07-22

Accepted: 2018-09-02

UDC: 616.1

J Clin Med Kaz 2018;3(49):15-23

Corresponding Author: Assem Nogaibayeva,

Extracorporeal Hemocorrection Laboratory,

National Research Cardiac Surgery Center, Astana,

Republic of Kazakhstan. Tel.: +77013958158

E-mail: assemkatolegen@gmail.com

\section{Abstract}

Pathophysiological, pathomorphological and functional changes in the kidneys after left ventricular assist device implantation have not yet been fully understood and represent an actual problem of our time. Changes in renal function following left ventricular assist device implantation have been disclosed in many studies with inconsistent results. Some studies have shown that patients with renal dysfunction after left ventricular assist device implantation had worse outcomes, despite stabilization or even improvement of kidney function during the postoperative period, other studies have shown the progression of chronic kidney disease requiring renal replacement therapy.

Key words: kidney, left ventricular assist device

\section{СОЛ ЖАҚ ҚАРЫНШАНЫН ҚОСЫМША ҚАНАЙНАЛЫМ ЖУЙЕСІН ИМПЛАНТАЦИЯЛАУ ЖӘНЕ БҮЙРЕК ФУНКЦИЯСЫНЫН БҰЗЫЛУЫ}

Ноғайбаева Ә.Т. ${ }^{1}$, Султанова Б.Ғ. ${ }^{2}$, Махмутов М.Б. ${ }^{3}$

${ }_{1}$ Экстракорпоралдық гемотүзету зертханасы, Ұлттық ғылыми кардиохирургия орталығы, Астана, Қазақстан Республикасы

${ }^{2}$ Нефрология және гемодиализ кафедрасы, Қазақ медициналық үздіксіз білім беру университеті, Алматы, Қазақстан Республикасы

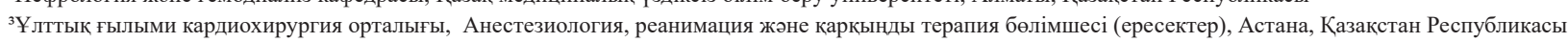

\section{ТҰЖЫРЫМДАМА}

Сол жақ қарыншалық айналымға арналған көмекші құрал имплантациядан кейін патофизиологиялық, морфологиялық және фрункционалдық бүйрек патологиялық өзгерістер әлі толық түсінген және бүгінгі күні өзекті мәселе болып табылады емес. Сол жақ қарыншалық айналымға арналған көмекші құрал имплантациядан кейін бүйрек функциясының өзгерістер қайшылықты нәтижелері көптеген зерттеулерде анықталды. Кейбір зерттеулер, бүйрек қызметі бұзылған науқастарда операциядан кейін кезеңде тұрақтандыруға немесе бүйрек функциясының жақсаруына қарамастан нашар нәтижені көрсетті, басқа зерттеулер бүйрек ауыстыру терапиясын қажет ететін созылмалы бүйрек ауруларының прогрессиясын көрсетті.

Негізгі сөздер: бүйрек, сол жақ қарыншаның қосалқы қанайналым жүйесі

\section{ИМПЛАНТАЦИЯ ВСПОМОГАТЕЛЬНОГО УСТРОЙСТВА КРОВООБРАЩЕНИЯ ЛЕВОГО ЖЕЛУДОЧКА И ПОЧЕЧНАЯ ДИСФУНКЦИЯ}

\section{Ногайбаева А.Т. ${ }^{1}$, Султанова Б.Г.², Махмутов М.Б. ${ }^{3}$}

'Лаборатория экстракорпоральной гемокоррекции, Национальный научный кардиохирургический центр, Астана, Республика Казахстан

${ }^{2}$ Кафедра нефрологии и гемодиализа, Казахский Медицинский Университет непрерывного образования, Алматы, Республика Казахстан

${ }^{3}$ Отделение анестезиологии, реанимации и интенсивной терапии (взрослых), Национальный научный кардиохирургический центр, Астана, Республика Казахстан

\section{PEЗЮME}

Патофизиологические, патоморфологические и функциональные изменения почек после имплантации вспомогательного устройства кровообращения левого желудочка до сих пор до конца не изучены и представляет собой актуальную проблему современности. Изменения почечной функции после имплантации вспомогательного устройства кровообращения левого желудочка были раскрыты во многих исследованиях с противоречивыми результатами. Некоторые исследования показали, что пациенты с почечной дисфункцией, после имплантации вспомогательного устройства кровообращения левого желудочка, имели более худший исход, несмотря на стабилизацию или даже улучшение работы почек в послеоперационном периоде, другие исследования показали прогрессирование хронической болезни почек, потребовавшей заместительной почечной терапии.

Ключевые слова: почки, искусственный левый желудочек 


\section{Введение}

Сердечная недостаточность $(\mathrm{CH})$ - прогрессирующее заболевание, являющееся экономическим бременем для современного здравоохранения. СН, в свою очередь достигла размеров глобальной пандемии, затрагивающей по меньшей мере 26 миллионов человек во всем мире и увеличивающаяся в распространенности [1]. Так, по последним данным 2018 года Американской Ассоциации кардиологов: сердечнососудистые заболевания, перечисленные в качестве основной причины смерти, составляют около 836546 смертей в США, являются ведущей мировой причиной смертности, в результате чего, в 2015 году доля составила более 17,9 миллионов смертей в год, а по прогнозам, возрастет до 23,6 миллионов человек к 2030 году. Ожидаемые общие прямые медицинские расходы на кардиоваскулярную патологию увеличатся до 749 миллиардов долларов США к 2035 году [2]. Расходы на здравоохранение при СН значительно возрастут со старением населения. В настоящее время у 5,7 миллиона человек в США имеется СН, к 2030 году ожидается, что более чем 8 миллионов человек будут страдать от этого состояния, что обуславливает увеличение распространенности на 46\% [3].

$\mathrm{CH}$ часто характеризуется хронической почечной дисфункцией (ПДф). Наличие как $\mathrm{CH}$, так и почечной недостаточности не только усложняет контроль за состоянием пациентов, но также способствует увеличению заболеваемости и смертности. Распространенность умеренной и тяжелой почечной недостаточности, определяемая как скорость клубочковой фильтрации (СКФ) менее 60 мл/мин/1,73 м2, нормальная - более 90 мл/мин/1,73 м2, составляет примерно 30-60\% у пациентов с СН. К примеру, в рамках систематического обзора 16 исследований, включившего более 80000 госпитализированных и не госпитализированных пациентов с СН, у 29\% пациентов отмечались признаки умеренной и тяжелой почечной недостаточность (определяемой как расчетная СКФ менее 53 мл/мин, креатинин сыворотки 1,5 мг/дл (132 мкмоль/л) [4].

По данным Hasin Т. и соавт., в случае хронической сердечной недостаточности (ХCH) умеренная и тяжелая почечная дисфункция (СКФ менее 60 мл/мин/1,73 м2) была зарегистрирована у 45\% амбулаторных пациентов с $\mathrm{XCH}$ и у $64 \%$ пациентов, госпитализированных по поводу декомпенсированной сердечной недостаточности. По сравнению с пациентами с сердечной недостаточностью без нарушения функции почек среди этих пациентов встречается более высокий уровень заболеваемости и смертности [5].

Нарушение работы почек у пациентов с тяжелой ХСН вызывает интерес нефрологов, ввиду сложности дифференцирования обратимых нарушений на фоне кардиоренального синдрома 2 типа с состоявшейся хронической болезнью почек (ХБП), развития тяжелых гемодинамических осложнений при необходимости взятия на заместительную почечную терапию (например, гипотензия, потребность в вазопрессорах).

Вопрос о почечной функции особенно важен у тех пациентов с терминальной стадией сердечной недостаточности, которых готовят для имплантации вспомогательного устройства левого желудочка (Left Ventricular Assist Device - LVAD), выступающего в качестве стандарта лечения [6] или трансплантации сердца. С 2013 года в Республике Казахстан (РК) внедрен клинический протокол диагностики и лечения «Хирургическое лечение хронической сердечной недостаточности - имплантация вспомогательных устройств (искусственные желудочки сердца (LVAD, RVAD, BiVAD), искусственного сердца (TAH) и трансплантация донорского сердца». В ближайшем будущем планируется передача операции - имплантации LVAD в регионарные кардиохирургические центры. При этом своевременная диагностика почечной дисфункции, a также постимплантационный мониторинг, позволят предупредить переход в ХБП 3 стадии, удлинить додиализный период и таким образом отсрочить начало дорогостоящей заместительной почечной терапии у данной категории пациентов.

В случае развития необратимых нарушений функции почек у пациентов с LVAD, ожидающих трансплантации сердца, может потребоваться решение вопроса о симультанной трансплантации сердце-почка. Необходимо отметить, более высокий риск развития и прогрессирования почечной недостаточности у реципиентов сердца в посттрансплантационном периоде, в сравнении с другими видами кардиохирургических вмешательств. В дальнейшем, риск связан с постоянным приемом иммунносупрессора - ингибитора кальциневрина, что также приводит к прогрессирующему снижению функции почек.

Следовательно, при кардиоренальном синдроме 2 типа, а также после имплантации LVAD, необходим обязательный тщательный мониторинг почечной функции, это в свою очередь позволит своевременно предупредить развитие острого почечного повреждения и сделать прогноз в отношении почек в посттрансплантационном периоде.

Как правило, предоперационная оценка функции почек у кандидатов на непочечную трансплантацию солидных органов, должна быть сосредоточена на оценке анамнеза повреждения и дисфункции почек (включая продолжительность и обратимость), степени текущей функции почек, а также вероятность и скорость прогрессирования после трансплантации. Пациенты с вероятной 4 или 5 стадией хронической болезни почек (ХБП), до и после непочечной трансплантации солидного органа, а также пациенты с установленной первичной почечной патологией, должны планироваться на симультанную трансплантацию органов (например, сердце+почка). В эпоху возрастающего дефицита органов и более длительного времени ожидания, вопрос о симультанной трансплантации неренальной и почечной должен быть тщательно рассмотрен. Подавляющее большинство пациентов с нормальным или умеренным нарушением функции почек должна планироваться только неренальная трансплантация солидных органов [7].

\section{Кардиоренальный синдром и терапия LVAD}

Патофизиология почек во время поддержки LVAD до сих пор до конца не изучена и представляет собой актуальную проблему современности. Изменения почечной функции после имплантации LVAD были раскрыты во многих исследованиях с противоречивыми результатами. Некоторые исследования показали, что пациенты с почечной дисфункцией после имплантации LVAD имели более худший исход, несмотря на стабилизацию или даже улучшение работы почек в послеоперационном периоде [5]. 
Другие исследования показали прогрессирование почечной дисфункции, потребовавшей временной заместительной почечной терапии или программного гемодиализа. В ретроспективном одноцентровом исследовании Verma S. и соавт., не было выявлено достоверно значимой разницы в частоте смертности, развития ОПП, потребности в заместительной почечной терапии и изменения средней СКФ в течение 6 месяцев, при сравнительной оценке группы пациентов после имплантации LVAD с группой пациентов на инотропной поддержке, ожидавших трансплантации сердца [8].

Новые модели вспомогательных устройств кровообращения, более тщательный отбор пациентов, позволят сократить уровень смертности, острого почечного повреждения (ОПП), а также переход в хроническую болезнь почек (ХБП) или прогрессирование уже имеющейся патологии почек. В связи с чем, нужна максимально точная оценка клинико-лабораторных параметров пациента, которые смогут точно спрогнозировать исход после имплантации LVAD, важна для оптимального выбора терапевтических опций и уменьшения послеоперационного риска.

Тяжелая ПДф (программный гемодиализ или креатинин сыворотки крови более 2,5 мг/дл) считается относительным противопоказанием к имплантации LVAD [9].

Однако, наличие ПДф до имплантации LVAD не обязательно может быть сильным предиктором развития острого почечного повреждения после имплантации. Вопрос обратимости нарушения функции почек при кардиоренальном синдроме является серьезной проблемой у пациентов, подлежащих трансплантации сердца.

Существует множество важных взаимодействий между заболеванием сердца и заболеванием почек. Данное взаимодействие носит двунаправленный характер, так как острая, так и хроническая дисфункции сердца или почек могут спровоцировать острое или хроническое нарушение функции другого органа. Клиническая значимость данных взаимоотношений отражена в следующих наблюдениях:

- смертность возрастает у пациентов с ХСН при сниженной СКФ.

- пациенты с ХБП находятся в группе высокого риска и сердечно-сосудистых событий и сердечной недостаточности, при этом сердечно-сосудистые события обусловливают $50 \%$ смертности у пациентов с почечной недостаточностью.

- острые или хронические системные нарушения могут обусловить как сердечную, так и почечную дисфункции [10].

Термин «кардиоренальный синдром» (КРС) был предложен для данных взаимодействий, но определение и классификация до сих пор остаются не уточненными.

Клаудио Ронко и соавторами была предложена классификация:

- КРС 1 типа (острый) - острая сердечная недостаточность приводит к острому почечному повреждению (ОПП);

- КРС 2 типа - хроническая сердечная дисфункция/ ХСН обусловливает прогрессирующую ХБП/ХПН;

- КРС 3 типа - резкое и первичное ухудшение функции почек, вызванное, например, почечной ишемией или гломерулонефритом, вызывает острую сердечную дисфункцию, которая может проявиться в виде СН;

- КРС 4 типа - первичная ХБП приводит к сердечной дисфукнции, которая может манифестировать коронарной болезнью сердца, ХСН или аритмией;

- КРС 5 типа (вторичный) - острое или хроническое системной заболевание (например, сепсис, васкулит, амилоидоз или сахарный диабет), которое обусловливает одновременно сердечную и почечную дисфункции [11].

Таким образом, наибольший интерес в ситуации изменением работы почек после имплантации LVAD, представляет кардиоренальный синдром 2 типа. Что в свою очередь, требует тщательного анализа «почечных» эффектов LVAD, с точки зрения гемодинамики, физиологии, патологии и клинических проблем до и после имплантации устройств, чтобы помочь выявить пациентов, наиболее подходящих для использования данного вида терапии, а также уточнить терапию для снижения ассоциированных рисков.

\section{Описание механизма LVAD и подбор паци- ентов к имплантации LVAD}

Вспомогательные устройства кровообращения желудочков с непрерывным потоком (используемые главным образом как вспомогательные устройства для левого желудочка [LVAD] и реже для бивентрикулярной поддержки [BiVAD]) все чаще используются для лечения пациентов c сердечной недостаточностью на конечной стадии (т.е. на стадии D рефрактерной сердечной недостаточности, требующей специализированных вмешательств), как в качестве моста для трансплантации, временной терапии при острых состояниях, так и в качестве «целевой терапии» (известной как LVAD-Destination Therapy) для пациентов, не являющихся кандидатами на пересадку сердца из-за преклонного возраста или других медицинских сопутствующих заболеваний. И таким образом, выступают в качестве стандарта терапии пациентов с терминальной $\mathrm{XCH}$ [12]. Послеоперационный курс осложняется частыми госпитализациями из-за кровотечений, инфекций, тромбоза насоса, сердечной недостаточности и неисправностях устройства, которые требуют надлежащего внимания и управления.

Искусственный желудочек сердца - (англ. VAD - Ventricular Assist Device) - механическое устройство, частично или полностью заменяющее функцию сердца при сердечной недостаточности. Левожелудочковая система вспомогательного кровообращения (англ. LVAD - Left Ventricular Assist Device) - медицинское устройство для обеспечения терапевтического действия для лиц с прогрессирующей сердечной недостаточностью, полностью или частично замещающее функции левого желудочка сердца, восстанавливая общую перфузию у пациента и облегчая внутреннюю патологическую симптоматику [13].

Принцип работы искусственных желудочков сердца заключается в механической разгрузке левого или правого желудочка сердца. Канюля имплантируется в верхушку желудочка, далее кровь через насос вспомогательного устройства идет в канюлю, имплантируемую в аорту. Кровь из желудочка попадает в приточную канюлю вдоль центральной оси и под правильными углами выталкивается лопастями ротора и между ними. Вращение ротора производится вокруг центральной оси. Жидкость, получившая таким образом ускорение, собирается и перемещается вокруг спиральной камеры, а затем выводится под нужным давлением и с нужной скоростью путем направления в отточную канюлю по касательной. Ротор 
полностью находится на магнитной подвеске, что в свою очередь позволяет избежать использовать механические подшипники и его изнашиваемости [13].

Подбор пациента и сроки имплантации являются основными главными определяющими факторами успеха терапии LVAD. Пациентов оценивают по (1) соответствию для вспомогательного кровообращения с помощью LVAD, базирующемуся на степени заболевания, (2) способности успешно перенести операцию и (3) возможности выписаться домой с адекватной поддержкой семьи/сиделки для долгосрочного успеха. Одним из ключевых моментов в отборе пациентов является раннее применение имплантации для предотвращения прогрессирующей недостаточности органа-мишени. Определение пригодности пациента для установки LVAD и сроков проведения имплантации устройства требуют тщательного сопоставления возможных рисков и преимуществ, профили пациентов и предположительные сроки указаны в Таблице 1 [14].

Таблица 1 Профили пациента по классификации INTERMACS (Межведомственный регистр случаев тяжелой сердечной недостаточности, при которой показано применение механических устройств для вспомогательного кровообращения) и временные рамки начала механической поддержки кровообращения.

\begin{tabular}{|c|c|c|}
\hline Профиль № & Описание & $\begin{array}{l}\text { Время до механической поддержки кровоо- } \\
\text { бращения }\end{array}$ \\
\hline 1 & Критический кардиогенный шок. & В пределах нескольких часов \\
\hline 2 & $\begin{array}{l}\text { «Прогрессирующее ухудшение» - зависимость от введения препаратов поло- } \\
\text { жительного инотропного действия (ППИД) с непрерывным ухудшением. }\end{array}$ & В пределах нескольких дней \\
\hline 3 & $\begin{array}{l}\text { «Стабильны, но зависимы от введения ППИД» - описывает клиническую } \\
\text { стабильность при введении от малых до средних доз внутривенных ППИд (в } \\
\text { данный профиль не входят пациенты с временной поддержкой кровообраще- } \\
\text { ния без введения ППИд). }\end{array}$ & В пределах нескольких недель \\
\hline 4 & $\begin{array}{l}\text { «Рецидивирующая прогрессирующая сердечная недостаточность» - «рециди- } \\
\text { вирующая» скорее, чем «рефрактерная» декомпенсация. }\end{array}$ & В пределах недель-месяцев \\
\hline 5 & $\begin{array}{l}\text { «Непереносимость физических нагрузок» - описывает пациентов, которые } \\
\text { комфортно чувствуют себя в покое, но не переносят физической нагрузки. }\end{array}$ & Варьирует \\
\hline 6 & $\begin{array}{l}\text { «Физические нагрузки ограничены» - описывает пациента, который способен } \\
\text { выполнять определенную легкую работу, но усталость наступает через не- } \\
\text { сколько минут после какой-либо значительной физической нагрузки. }\end{array}$ & Варьирует \\
\hline 7 & $\begin{array}{l}\text { «Прогрессирующая III стадия по NYHA» - описывает пациентов, которые кли- } \\
\text { нически стабильны с умеренным уровнем комфортной активности, несмотря } \\
\text { на предшествующую, но не недавнюю декомпенсацию в анамнезе. }\end{array}$ & $\begin{array}{l}\text { Не является кандидатом для механической } \\
\text { поддержки кровообращения }\end{array}$ \\
\hline
\end{tabular}

\section{Экспериментальные работы по воздей- ствию LVAD на функцию почек}

Начиная с 1980x гг. начинают публиковаться статьи по экспериментальным работам со вспомогательными устройствами левого желудочка (LVAD). Ввиду того, что почечная функция является предиктором исхода после имплантации LVAD, а развитие острого почечного повреждения часто связано с необходимостью переливания нескольких доз крови, в течение интра или послеоперационного периодов, проблема почечной дисфункции у данной группы пациентов привлекла внимание исследователей.

В первую очередь начала изучаться проблема гемодинамических воздействий на локальный почечный кровоток, создаваемого LVAD. Так, после имплантации LVAD, у собак с индуцированным кардиогенным шоком, без инотропной поддержки, перфузия во всех органах нормализовалась, за исключением почечного кровотока, оставшегося угнетенным примерно на уровне 56\% от прешокового уровня. Даже в сочетании с инотропной поддержкой дофамином 5 мкг/кг/мин, уровень почечного кровотока достигал $83 \%$ от прешокового состояния. В заключении данного эксперимента, был сделан вывод, что имплантация LVAD в сочетании с низкими дозами инотропной поддержки может предупредить ухудшение перфузии органов после кардиогенного шока, исключая почки [16].

Ухудшение функции почек распространено у пациентов с прогрессирующей сердечной недостаточностью, кроме того, сниженная азотовыделительная функция также считается противопоказанием к сердечной трансплантации; однако этот вопрос до сих пор остается спорным. Вопервых, нет единогласно принятого уровня нарушения почечной функции, ниже которого трансплантация считается абсолютно противопоказанной, хотя многие центры требуют клиренса креатинина $(\mathrm{CrCl})$ не менее 50 мл/мин для проведения трансплантации. Это основано на исследованиях, показывающих более высокий риск посттрансплантационной смертности, ассоциируемый с прогрессирующей почечной недостаточностью. Что было подтверждено исследованием Ostermann и соавторов, 2004г., выявившим у пациентов с уровнем клиренса креатинина ниже 50 мл/мин до трансплантации сердца, увеличение риска смертности в 2 раза на 30 сутки, в сравнении с пациентами с предтрансплантационным клиренсом креатинина выше 50 мл/мин.

В свою очередь, в исследовании по изменению профиля биомаркеров и нейромедиаторов, Ahmad T. и соавторы обнаружили постоянные аномальные концентрации NGAL после имплантации LVAD, несмотря на нормализацию уровней креатинина в сыворотке, и это ставит под сомнение применение только лишь клиренса креатинина для качественного мониторинга острого почечного повреждения. Они также предположили, что прогрессирующая правожелудочковая недостаточность и системный венозный застой в условиях унивентрикулярной поддержки вероятно способствовали постоянному повреждению почечных канальцев [17].

В другом многоцентровом исследовании J. Batler и соавторы провели ретроспективный анализ изменения почечной функции у 220 пациентов с тяжелой сердечной Journal of Clinical Medicine of Kazakhstan: Volume 3, Number 49, Issue 2018 
недостаточностью, подвергшихся имплантации LVAD за период с 1996 по 2003 годы. Исследование подтвердило наличие ассоциации исходно низких показателей почечной функции с худшими исходами после имплантации LVAD. Тем не менее, это же исследование обнаружило, что функция почек значительно и быстро улучшается у выживших пациентов после имплантации LVAD и ассоциируется с хорошим исходом.

В данном исследовании пациенты были разделены на 4 группы на основании квартилей исходного клиренса креатинина $(<47,48-68,69-95$, and $>95)$. Затем данные группы сравнили по исходам постимплантационного периода. Исследование показало, что выживаемость с LVAD оказалась хуже у пациентов с более низким исходным уровнем клиренса креатинина $(42 \%, 52 \%, 63 \%$ и $79 \%$ в течение 6 месяцев и 26\%,34\%, 47\% и 66\% в течение 12 месяцев выживаемости для 1-4 квартилей, при $\mathrm{p}<0.01)$. Для 60 пациентов с исходным клиренсом креатинина менее 50, клиренс креатинина возрос с 36.7 +/- 9.2 до 60.1 +/35.5 ( $\mathrm{p}<0.01 ; \mathrm{n}=55$ пар) через неделю от имплантации. Восстановление почечной функции до клиренса креатинина более 50 было связано с тенденцией к лучшей 30-дневной выживаемости (84\% против 66\%, p =0,09) [18].

Таким образом, до сегодняшнего дня сведения о положительном и отрицательном влиянии имплантации на почечную функцию остаются противоречивыми.

С одной стороны, имплантация LVAD может улучшить работу почек и, следовательно, может улучшить прогноз; с другой стороны, нарушенная ренальная функция может увеличить риск неблагоприятных результатов после LVAD.

Более ранние исследования взаимосвязи между почечной дисфункциейирезультатами LVAD противоречивы. Это подтверждают результаты воздействия LVAD-системы Jarvik 2000, в котором критерии отбора исключали пациентов c почечной недостаточностью. В периоды поддержки кровообращения не отмечалось повышения креатинина, диурез также был сохранен. Почечная функция была хорошо сохранена в данной популяции пациентов. В заключение наши данные свидетельствовали о том, что Jarvik 2000 LVAD, имплантируемое непрерывное осевое устройство, обеспечило отличную физиологическую поддержку почек и печени в течение как минимум от 2 месяцев и до 6 месяцев после имплантации [19].

\section{Почечная гемодинамика и патофизиоло- гические изменения в почках после им- плантации LVAD}

Положительное влияние LVAD на работу почек предположительно обусловлено, не только улучшением гемодинамики, вследствие восстановления сердечного выброса и улучшения перфузии органов [20], но и уменьшением вредных негемодинамических факторов, таких как активация симпатической нервной системы (отмечалось снижение уровня эпинефрина, норэпинефрина), гиперактивность ренин-ангиотензинальдостероновой системы (снижались активность ренина плазмы, уровень ангиотензина II, вазопрессина и предсердного натриуретического пептида, в том числе) и инактивация оксида азота, то есть ряда факторов, которые, как известно, ответственны за почечную дисфункцию при сердечной недостаточности [21].

\section{Улучшение перераспределения почечного кровотока}

Во время кардиогенного шока или низкого сердечного выброса, почечный артериальный кровоток, главным образом кортикальный кровоток, уменьшается, когда давление перфузии ниже критического уровня, что приводит к перераспределению почечного кровотока. В исследовании Flaherty M.P. et al. подтвердился нефропротективный эффект имплантированного перкутанного LVAD, в группе которого отмечалось уменьшение развития ОПП у пациентов с ХБП и низкой фракцией выброса после чрескожных коронарных интервенций [22].

B ранних экспериментальных исследованиях, Sezai et al. Было обнаружено, что почечный кортикальный кровоток увеличивался, но кровоток мозгового вещества снижался в случае применения LVAD с пульсативным кровотоком в острой модели инфаркта миокарда у свиней. Nemoto сравнивал влияние пульсативного и непульсативного VAD на почечный кровоток при кардиогенном шоке у свиней, и различия между группами с искусственной циркуляцией высокого уровня (скорость вспомогательного устройства $80 \%$ ) и низкого уровня (скорость вспомогательного устройства 60\%). При поддержке вспомогательного устройства перераспределение почечного кровотока и тканевого метаболизма улучшалось в разной степени, однако почечная артериальная кровь, почечные кортикальномозговые кровотоки и почечный тканевой кровоток были, по-видимому, превосходящими у пульсативных LVAD по сравнению с постоянным потоком, особенно при низких скоростях искусственной циркуляции. Исследование показало, что применение непульсативного LVAD при кардиогенном шоке будет более эффективным, в случае поддержания достаточного кровотока, по крайней мере $80 \%$ или более выше уровня аортального кровотока [23]. Nagasaka выявил, что сочетанное применение внутриаортальной баллонной контрапульсации и имплантации LVAD при правильно подобранных режимах, могут способствовать улучшению почечной функции [24]. Необходимость поддержания адекватной скорости кровотока подтверждается исследованием Miyama, при котором производилась регистрация уровня кислорода и метаболических сдвигов в крови почечной, верхней брыжеечной вены и легочной артерии: снижение кровотока приводило к гипоксемии, тяжелым метаболическим нарушениям и развитию ишемии органов у свиней. В 2012 году Iwashima et al. исследовали внутрипочечный кровоток у пациентов с имплантированным пульсативным LVAD, используя дуплексную допплеровскую сонографию. Имплантация сопровождалась значительным улучшением функции почек, уменьшением средней систолической скорости $(38,2 \pm 8,9$ до $28,3 \pm 2,2$ см/сек, $<00,05)$ и повышением средней конечной диастолической скорости (от $8,3 \pm 3,2$ до $11,3 \pm 1,3 \mathrm{~cm} /$ сек, $\mathrm{p}<0,05)$ и, как следствие, значительным улучшением среднего почечного резистивного индекса $(0,79$ $\pm 0,06-0,60 \pm 0,04, p<0,01)$. Улучшение функции почек могло быть частично обусловлено улучшением внутрипочечной гемодинамики [25].

\section{Влияние на активность ренин-ангиотен- зин-альдостероновой системы (РАAC)}

Исследования на животных с использованием моделей здорового сердца показали существенно повышенный уровень ангиотензина II в плазме в группе LVAD в сравнении 
с другими группами: $350 \pm 139$ LVAD, $8 \pm 6$ RVAD (Right Ventricular Assist Device - устройство вспомогательного кровообращения правого желудочка) и $3 \pm 2$ TAH (Total Artificial Heart - искусственное сердце) и $3 \pm 2$ группа контроля $(\mathrm{p}<0,001)$; а также повышенную активность ангиотензин-конвертирующего фермента: 325 \pm 59 LVAD, $6 \pm 4$ RVAD, $6 \pm 5$ TAH и $3 \pm 1$ группа контроля $(\mathrm{p}<0,001)$, дополнительные исследования показали, что уменьшенная пульсация, возникающая при имплантации с непрерывным потоком LVAD, вызвала сильный периартериит в почках, местная система ренинангиотензина была активирована в воспалительных клетках только в группе непрерывного потока LVAD [26].

James et al. также обнаружили, что у пациентов, ожидающих трансплантации, активность ренина и альдостерона плазмы значительно снизилась по сравнению с исходным уровнем, на 4 и на 8 неделях после имплантации LVAD. Кроме того, снижение активности PAAC произошло раньше, чем снижение объема плазмы и уровня предсердного натрийуретического пептида плазмы, что указывает на улучшение сердечной функции посредством LVAD, что было связано с нормализацией объемной нагрузки, вторичной по отношению к нормализации ее регуляторных веществ [27].

По последним данным Grosman-Rimon L. et al 2018 г., уровни нейрогормонов оценивавшиеся у реципиентов LVAD с непрерывным потоком сравнивали с пациентами с $\mathrm{CH}$ и с группой контроля (здоровые лица). В результате уровни альдостерона, норэпинефрина и ренина были повышены в такой же степени у реципиентов LVAD и пациентов с CH по сравнению со здоровыми лицами группы контроля. Выводы о том, что альдостерон, норадреналин и ренин были повышены после восстановления гемодинамических функций при поддержке LVAD, свидетельствуют о том, что уровни нейрогормонов не нормализуются [28].

\section{Влияние на симпатическую нервную си- стему}

Хотя подавление симпатической активности является важной задачей для текущей стратегии лечения сердечной недостаточности, мало что известно о взаимосвязи между установкой скорости вращения и вегетативной нервной деятельностью при непрерывном лечении LVAD. Автономная деятельность может варьироваться в зависимости от скорости вращения. У пациентов с более высокой скоростью вращения лучше подавлялась симпатическая активность [29].

Markham DW et al, 2013 г., обнаружил, что у пациентов с сердечной недостаточностью с непрерывными, непульсативными LVAD, отмечается симпатическая активация, что, вероятно, частично связано, с разгрузкой барорецепторов. Такая хроническая симпатическая активация может способствовать или ухудшать патологию органов-мишеней органов и уменьшать вероятность эксплантации желудочков. Для достижения оптимальных результатов у этих пациентов могут потребоваться стратегии для обеспечения минимальной степени артериальной пульсации, даже при непрерывном течении LVAD [30].

\section{Патологические изменения}

Ambardekar A.V. et al обнаружили, что после воздействия непрерывного потока LVAD морфологически отмечалось увеличение толщины стенки аорты, коллагена и гладкомышечного содержимого, сопровождающееся уменьшением содержания эластина и муцинового основного вещества, по сравнению с мофологическими изменениями у пациентов с $\mathrm{CH}$, также кривые стресс-деформации аорты выявили повышенную жесткость сосуда в группе $\mathrm{CH}+$ LVAD по сравнению с группой СН [31].

Hasin T. и соавт. у пациентов, наблюдавшихся после имплантации HeartMate II, отметил развитие артериосклероза в посмертных почечных образцах, а также снижение СКФ через год от предимплантационного уровня [32].

Как видно из перечисленных экспериментальных и клинических работ, данные по влиянию имплантации LVAD на физиологию и морфологические изменения сосудов почек до сих пор весьма противоречивы.

\section{Факторы риска и клинические исходы по- сле имплантации LVAD}

Начиная с 2013 года возросло число клинических исследований по определению факторов риска выживаемости, прогноза и динамических изменений почечной функции после имплантации LVAD.

Mohamedali B. и Bhat G. подтверждают, что СКФ до имплантации является важным прогностическим маркером, поскольку пациенты с СКФ<60 мл/мин подвергаются более высокому риску сердечно-сосудистой заболеваемости и смертности. Пациенты с базовой СКФ $<60$ мл/мин имели более высокую смертность от всех причин, чем у пациентов с предимплантационной СКФ $\geq 60$ мл/мин (соответственно $45 \%$ и $27 \%, \mathrm{p}=0,006)$. У пациентов с низкой СКФ чаще развивалась правожелудочковая недостаточность, анализ выживаемости Каплана-Мейера подтвердил низкую выживаемость в этой группе [33]. В целом показателями, связанными с плохими исходами, являются уровень креатинина более 2,5 мг/дл, уровень мочевины крови более 40 мг/дл или расчетная СКФ менее 0,5 мл/кг/мин. Диурез ниже 20-30 мл/час в течение 6-8 часов также является фактором риска ухудшения функции почек и смерти в послеоперационном периоде [15].

Неоднократно проводилось изучение работы почек у пациентов с постоянным потоком LVAD. В когорте пациентов с непрерывным потоком LVAD, поддерживаемых в течение по крайней мере одного года, уровни креатинина и мочевины первоначально снижались, а затем постепенно увеличивались в течение следующего года, а значения в течение одного года оставались значительно ниже их исходных значений [34]. В других исследованиях, которые непосредственно сравнивали пульсационные и непрерывные LVAD, в качестве исходов использовали азот мочевины в сыворотке, креатинин, клиренс креатинина и скорость клубочковой фильтрации. Они единообразно продемонстрировали, что между двумя группами не было различий в функции почек. Напротив, рандомизированная контролируемая оценка устройств с непрерывным и пульсирующим потоком для использования в терапии назначения показала значительно меньшую скорость постимплантационной почечной недостаточности у пациентов с устройствами с непрерывным потоком [35]. Использование механических устройств кровообращения приводит к улучшению функции почек у пациентов после имплантации, однако почечные исходы после трансплантации сердца, по-видимому, в большей степени зависят от уровня почечной функции, достигнутого во время имплантации LVAD, чем от базового уровня функции почек [36]. При 
этом в случае экстренной имплантации LVAD, несмотря на то, что у пациентов отмечались более высокая частота послеоперационной правожелудочковой недостаточности и более худшие предоперационные показатели функции печени и почек, в целом была отмечена сходная картина выживаемости среднего возраста и аналогичная частота других послеоперационных осложнений [37].

В ретроспективном многоцентровом когортном исследовании Muslem et al в качестве предикторов неблагоприятного прогноза рассматривались возраст и СКФ, в результате было выявлено, что пожилой возраст, низкий уровень СКФ и 1 класс INTERMACS оказались независимыми предикторами 1-летней смертности. Более того, пожилые пациенты (возраст> 60 лет) с нарушенной функцией почек (СКФ $<55$ мл/мин/1,73 м2) имели 5-кратное увеличение риска смертности в течение первого года после имплантации $(\mathrm{p}<0,001)$. В заключении было отмечено, что возраст старше 60 лет является независимым предиктором нарушения функции почек и смертности, а пожилой возраст в сочетании со сниженной почечной функцией до имплантации имел кумулятивное отрицательное влияние на выживаемость у пациентов, получавших терапию LVAD [38]. При этом в случае тщательного подбора пациентов, лица старше 65 лет, получавших терапию LVAD с постоянным потоком, демонстрировали хороший уровень выживаемости [39]. В японском национальном исследовании, при сравнении группы лиц молодого возраста, с лицами старше 60 лет, через 1 и 3 года выживаемость после имплантации LVAD составляла 95\%, 91\% у более молодых пациентов и $85 \%$, у 75\% пожилых пациентов соответственно $(\mathrm{P}=0,016)$, хотя возраст не был фактором риска для многомерного анализа, однако, улучшение функции почек после 60 лет было временным, и улучшение длилось не более, чем 3 месяца [40].

Из-за нехватки донорских сердец и нынешнего алгоритма трансплантации сердца поддержка LVAD для кандидатов на пересадку всех возрастных групп, вероятно, приведет к расширению списка очередности и длительному использованию LVAD со значительными бюджетными последствиями, но без увеличения количества пересадки сердца. Факты с ограниченным уровнем 4 показали, что поддержка LVAD у детей приводила к выживаемости, сравнимой с показателями взрослого населения.

Кроме этого, кардиологам необходимо рассмотреть вопросы более ранней имплантации LVAD, на этапе относительно удовлетворительной функции органов, для предотвращения прогрессирующего ухудшения работы органов -мишеней, так как последующее улучшение перфузии почек может уменьшить частоту послеоперационного ОПП и привести к улучшению долгосрочной ренальной функции и улучшенным отдаленным результатам. Использование технологии, поддерживающей LVAD, может превратить долю пациентов, не подлежащих трансплантации сердца, в приемлемых кандидатов, вследствие благоприятного влияния на функцию конечных органов, включая восстановление почечной функции. Дальнейшие исследования необходимы, чтобы помочь выявить пациентов, наиболее подходящих для использования этой технологии, и уточнить терапию для снижения связанных с ней рисков.

Применение имплантируемых вспомогательных устройств левого желудочка все чаще используются у пациентов с сердечной недостаточностью на конечной стадии, невосприимчивой к традиционной медикаментозной терапии. В свете растущего числа пациентов, с поддержкой LVAD как в Республике Казахстан, так и по всему миру, в качестве терапии назначения, важно понять влияние этих устройств на почки. Для лучшего понимания взаимосвязи между LVAD и почкой необходимы дополнительные исследования и результаты долгосрочных наблюдений. Так, при достаточном количестве наблюдений, планируется выявление факторов риска развития и прогрессирования ХПБ 3 стадии, ОПП в послеоперационном периоде, с помощью бинарной логистической регрессии. С помощью статистической модели линейной регрессии при параллельно набранном количестве пациентов с терминальной сердечной недостаточностью на консерватином лечении, возможен будет сравнительный анализ почечной функции при ХСН.

Таким образом, необходимо продолжить развивать стратегию скрининга и усовершенствования мер профилактики почечной дисфункции, выделения групп риска с ранним назначением нефропротективной терапии, а своевременная имплантация LVAD до развития необратимых процессов декомпенсации в органах-мишенях, позволит замедлить прогрессирование ХБП, удлинить додиализный период.

Вопрос о сроке поддержания функции почек в норме в постимплантационном периоде остается открытым, поскольку пациенты с диализ-потребным ОПП, могут со временем перейти в категорию пациентов с ТХПН на программном гемодиализе. Поэтому вопросы о других видах заместительной почечной терапии таких как, перитонеальный диализ, домашний диализ, должны быть рассмотрены в качестве альтернативы, кроме того, необходимо, решить вопросы о сроках формирования артерио-венозной фистулы, проблемы управления и мониторинга гемодинамики и объема жидкости, уточнить и разработать отдельные протоколы контроля за ренальной анемией, гемодиализа, вопросы ухода за такими пациентами. А учитывая необходимость постоянного приема варфарина - откорректировать алгоритм управления антикоагуляцией, в связи с чем, и необходимы дальнейшие исследования в этой области.

Disclosures: There is no conflict of interest for all authors.

\section{Список литературы}

1. Tseng CC, Ramjankhan FZ, de Jonge N, Chamuleau SA. Advanced Strategies for EndStage Heart Failure: Combining Regenerative Approaches with LVAD, a New Horizon? Front Surg. 2015;2:10. PubMed PMID: 25905105. PMCID: PMC4387859. Epub 2015/04/07. eng.

2. Benjamin EJ VS, Callaway CW, Chang AR, Cheng S, Chiuve SE, Cushman M, Delling FN, Deo R, de Ferranti SD, Ferguson JF FM, Gillespie C, Isasi CR, Jimenez MC, Jordan LC, Judd SE, Lackland D, Lichtman JH, Lisabeth L, Liu, S LC, Lutsey PL, Matchar DB, Matsushita K, Mussolino ME, Nasir K, O’Flaherty M, Palaniappan LP, Pandey, DK RM, Ritchey MD, Rodriguez CJ, Roth GA, Rosamond WD, Sampson UKA, Satou GM, Shah SH, Spartano NL, Tirschwell DL TC, Voeks JH, Willey JZ, 
Wilkins JT, Wu JHY, Alger HM, Wong SS, Muntner P. Heart Disease and Stroke Statistics_-2018 Update A Report From the American Heart Association. Circulation; 20.03.2018. p. e67-e492.

3. Bloom MW, Greenberg B, Jaarsma T, Januzzi JL, Lam CSP, Maggioni AP, et al. Heart failure with reduced ejection fraction. Nat Rev Dis Primers. 2017 Aug;3:17058. PubMed PMID: 28836616. Epub 2017/08/24. eng.

4. Smith GL, Lichtman JH, Bracken MB, Shlipak MG, Phillips CO, DiCapua P, et al. Renal impairment and outcomes in heart failure: systematic review and meta-analysis. J Am Coll Cardiol. 2006 May;47(10):1987-96. PubMed PMID: 16697315. Epub 2006/04/24. eng.

5. Hasin T, Topilsky Y, Schirger JA, Li Z, Zhao Y, Boilson BA, et al. Changes in renal function after implantation of continuous-flow left ventricular assist devices. J Am Coll Cardiol. 2012 Jan;59(1):26-36. PubMed PMID: 22192665. eng.

6. Hetzer R, Javier MFDM, Delmo Walter EM. Role of paediatric assist device in bridge to transplant. Ann Cardiothorac Surg. 2018 Jan;7(1):82-98. PubMed PMID: 29492386. PMCID: PMC5827126. eng.

7. Bloom RD. Renal function and nonrenal solid organ transplantation // http :// www . uptodate.com / contents / renal function - and - nonrenal - solid - organ - transplantation. 2016.

8. Verma S, Bassily E, Leighton S, Mhaskar R, Sunjic I, Martin A, et al. Renal Function and Outcomes With Use of Left Ventricular Assist Device Implantation and Inotropes in End-Stage Heart Failure: A Retrospective Single Center Study. J Clin Med Res. 2017 Jul;9(7):596-604. PubMed PMID: 28611860. PMCID: PMC5458657. Epub 2017/05/22. eng.

9. Lund LH, Matthews J, Aaronson K. Patient selection for left ventricular assist devices. Eur J Heart Fail. 2010 May;12(5):434-43. PubMed PMID: 20172939. Epub 2010/02/19. eng.

10. Kiernan MS, Udelson JE, Sarnak M. Cardiorenal syndrome: Definition, prevalence, diagnosis, and pathophysiology // http:// www.uptodate.com/contents/cardiorenal-syndromedefinition-prevalence-diagnosis-and-pathophysiology. 2018.

11. Di Lullo L, Bellasi A, Barbera V, Russo D, Russo L, Di Iorio B, et al. Pathophysiology of the cardio-renal syndromes types 1-5: An uptodate. Indian Heart J. 20172017 Mar - Apr;69(2):255-65. PubMed PMID: 28460776. PMCID: PMC5415026. Epub 2017/01/22. eng.

12. Soliman OII, Akin S, Muslem R, Boersma E, Manintveld OC, Krabatsch T, et al. Derivation and Validation of a Novel Right-Sided Heart Failure Model After Implantation of Continuous Flow Left Ventricular Assist Devices: The EUROMACS (European Registry for Patients with Mechanical Circulatory Support) Right-Sided Heart Failure Risk Score. Circulation. 2018 Feb;137(9):891-906. PubMed PMID: 28847897. Epub 2017/08/27. eng.

13. Left ventricular assist device. HeartMate III, Thoratec Corporation. User guide. HeartMate III 2017.

14. Stewart GC, Mehra MR. A history of devices as an alternative to heart transplantation. Heart Fail Clin. 2014 Jan;10(1 Suppl):S1-12. PubMed PMID: 24262348. Epub 2013/10/10. eng.

15. Slaughter MS, Meyer AL, Birks EJ. Destination therapy with left ventricular assist devices: patient selection and outcomes. Curr Opin Cardiol. 2011 May;26(3):232-6. PubMed PMID: 21430527. eng.

16. Sukehiro S, Flameng W. Effects of left ventricular assist for cardiogenic shock on cardiac function and organ blood flow distribution. Ann Thorac Surg. 1990 Sep;50(3):374-83. PubMed PMID: 2400257. eng.

17. Ahmad T, Wang T, O’Brien EC, Samsky MD, Pura JA, Lokhnygina Y, et al. Effects of left ventricular assist device support on biomarkers of cardiovascular stress, fibrosis, fluid homeostasis, inflammation, and renal injury. JACC Heart Fail. 2015 Jan;3(1):30-9. PubMed PMID: 25447345. Epub 2014/11/12. eng.

18. Butler J, Geisberg C, Howser R, Portner PM, Rogers JG, Deng MC, et al. Relationship between renal function and left ventricular assist device use. Ann Thorac Surg. 2006 May;81(5):1745-51. PubMed PMID: 16631666. eng.

19. Letsou GV, Myers TJ, Gregoric ID, Delgado R, Shah N, Robertson K, et al. Continuous axial-flow left ventricular assist device (Jarvik 2000) maintains kidney and liver perfusion for up to 6 months. Ann Thorac Surg. 2003 Oct;76(4):1167-70. PubMed PMID: 14530006. eng.

20. Ambardekar AV, Buttrick PM. Reverse remodeling with left ventricular assist devices: a review of clinical, cellular, and molecular effects. Circ Heart Fail. 2011 Mar;4(2):224-33. PubMed PMID: 21406678. PMCID: PMC3385856. eng.

21. Flaherty MP, Pant S, Patel SV, Kilgore T, Dassanayaka S, Loughran JH, et al. Hemodynamic Support With a Microaxial Percutaneous Left Ventricular Assist Device (Impella) Protects Against Acute Kidney Injury in Patients Undergoing High-Risk Percutaneous Coronary Intervention. Circ Res. 2017 Feb;120(4):692-700. PubMed PMID: 28073804. Epub 2017/01/10. eng.

22. Nemoto M. Experimental evaluation of the influence of complete artificial circulation on renal circulation and tissue metabolism - comparative study of pulsatile vs. nonpulsatile circulation. Ann Thorac Cardiovasc Surg 2003. p. 355-64.

23. Nagasaka F, Hasegawa T, Shiono M, Orime Y, Sezai Y. New sequential synchronized driving system of intraaortic balloon pumping and left ventricular assist device: influence on endocardial viability ratio and renal blood flow in their combination. Artif Organs. 1992 Apr;16(2):216-8. PubMed PMID: 10078249. eng.

24. Iwashima Y, Yanase M, Horio T, Seguchi O, Murata Y, Fujita T, et al. Effect of pulsatile left ventricular assist system implantation on Doppler measurements of renal hemodynamics in patients with advanced heart failure. Artif Organs. 2012 Apr;36(4):353-8. PubMed PMID: 21995604. Epub 2011/10/14. eng.

25. Ootaki C, Yamashita M, Ootaki Y, Kamohara K, Weber S, Klatte RS, et al. Reduced pulsatility induces periarteritis in kidney: role of the local renin-angiotensin system. J Thorac Cardiovasc Surg. 2008 Jul;136(1):150-8. PubMed PMID: 18603068. PMCID: PMC2533270. Epub 2008/05/19. eng.

26. Mao H, Katz N, Kim JC, Day S, Ronco C. Implantable left ventricular assist devices and the kidney. Blood Purif. 2014;37(1):5766. PubMed PMID: 24525434. Epub 2014/02/12. eng.

27. Grosman-Rimon L, McDonald MA, Freedman D, Yip P, Cherney DZ, Rao V. Neurohormone levels remain elevated in continuous flow left ventricular assist device recipients. J Card Surg. 2018 Jun. PubMed PMID: 29900585. Epub 2018/06/14. eng. 
28. Imamura T, Kinugawa K, Nitta D, Fujino T, Inaba T, Maki H, et al. Lower rotation speed stimulates sympathetic activation during continuous-flow left ventricular assist device treatment. J Artif Organs. 2015 Mar;18(1):20-6. PubMed PMID: 25337982. Epub 2014/10/22. eng.

29. Markham DW, Fu Q, Palmer MD, Drazner MH, Meyer DM, Bethea BT, et al. Sympathetic neural and hemodynamic responses to upright tilt in patients with pulsatile and nonpulsatile left ventricular assist devices. Circ Heart Fail. 2013 Mar;6(2):293-9. PubMed PMID: 23250982. Epub 2012/12/18. eng.

30. Ambardekar AV, Hunter KS, Babu AN, Tuder RM, Dodson RB, Lindenfeld J. Changes in Aortic Wall Structure, Composition, and Stiffness With Continuous-Flow Left Ventricular Assist Devices: A Pilot Study. Circ Heart Fail. 2015 Sep;8(5):944-52. PubMed PMID: 26136459. Epub 2015/07/01. eng.

31. Hasin T, Grupper A, Dillon JJ, Maleszewski JJ, Li Z, Topilsky Y, et al. Early Gains in Renal Function Following Implantation of HeartMate II Left Ventricular Assist Devices May Not Persist to One Year. ASAIO J. 2017 2017 Jul/Aug;63(4):401-7. PubMed PMID: 28114196. eng.

32. Mohamedali B, Bhat G. The Influence of Pre-Left Ventricular Assist Device (LVAD) Implantation Glomerular Filtration Rate on Long-Term LVAD Outcomes. Heart Lung Circ. 2017 Nov;26(11):1216-23. PubMed PMID: 28342643. Epub 2017/02/17. eng.

33. Miyamoto T, Karimov JH, Fukamachi K. Effects of continuous and pulsatile flows generated by ventricular assist devices on renal function and pathology. Expert Rev Med Devices. 2018 Mar;15(3):171-82. PubMed PMID: 29400088. Epub 2018/02/09. eng.

34. Slaughter MS, Rogers JG, Milano CA, Russell SD, Conte JV, Feldman D, et al. Advanced heart failure treated with continuousflow left ventricular assist device. N Engl J Med. 2009 Dec;361(23):2241-51. PubMed PMID: 19920051. Epub 2009/11/17. eng.

35. Singh M, Shullo M, Kormos RL, Lockard K, Zomak R, Simon MA, et al. Impact of renal function before mechanical circulatory support on posttransplant renal outcomes. Ann Thorac Surg. 2011 May;91(5):1348-54. PubMed PMID: 21524442. eng.

36. Tsiouris A, Morgan JA, Nemeh HW, Hodari A, Shah R, Brewer RJ, et al. Does elective or emergent operative status influence outcomes in patients undergoing implantation of left ventricular assist devices? Heart Surg Forum. 2014 Apr;17(2):E64-72. PubMed PMID: 24808443. eng.

37. Muslem R, Caliskan K, Akin S, Yasar YE, Sharma K, Gilotra NA, et al. Effect of Age and Renal Function on Survival After Left Ventricular Assist Device Implantation. Am J Cardiol. 2017 Dec;120(12):2221-5. PubMed PMID: 29037445. Epub 2017/09/20. eng.

38. Rosenbaum AN, John R, Liao KK, Adatya S, Colvin-Adams MM, Pritzker M, et al. Survival in elderly patients supported with continuous flow left ventricular assist device as bridge to transplantation or destination therapy. J Card Fail. 2014 Mar;20(3):1617. PubMed PMID: 24412524. Epub 2014/01/09. eng.

39. Yoshioka D, Toda K, Ono M, Nakatani T, Shiose A, Matsui Y, et al. Clinical Results, Adverse Events, and Change in EndOrgan Function in Elderly Patients With HeartMateII Left Ventricular Assist Device - Japanese Multicenter Study. Circ J. 2018 Jan;82(2):409-18. PubMed PMID: 29057766. Epub 2017/10/21. eng.

How to cite this article: Assem Nogaibayeva, Bagdat Sultanova, Myrzabek Makhmutov. Left Ventricular Assist Device (LVAD) Implantation and Renal Dysfunction [in Russian]. J Clin Med Kaz. 2018; 3(49):15-23 